\title{
Procesamiento de la información en la esquizofrenia: Amplitud de aprehensión $\left(^{*}\right)$
}

\section{José María Ruiz Vargas}

Universidad Autónoma de Madrid

Desde hace bastante tiempo, las anormalidades cognitivas, consideradas como una de las características básicas de la conducta esquizofrénica, han sido interpretadas como un fallo en alguna fase del procesamiento de la información entrante (Payne, 1960; MacGhie, 1966; Yates, 1966; Lawson, MacGhie y Chapman, 1967; Hawks y Marshall, 1971, entre otros). La amplitud de aprehensión, esto es, la medida de la cantidad de información a la que una persona puede atender simultáneamente, ha sido utilizada en algunas ocasiones para tratar de explicar el déficit esquizofrénico (Neale, McIntyre, Fox y Cromwell, 1969; Spohn, Thetford y Woodham, 1970; Cash, Neale y Cromwell, 1972), por considerarse una medida apropiada de la eficacia del procesamiento de la información, puesto que implica procesos atencionales, cognitivos y de memoria a corto plazo.

La amplitud de aprehensión es, pues, una medida de considerable relevancia para el enfoque del procesamiento de la información. Desde la invención del taquistoscopio, los experimentos sobre amplitud han utilizado generalmente letras que han sido expuestas por espacios tan cortos de tiempo que sólo permitían una fijación ocular. Los sujetos a los que se les pide que informen las letras que han visto, normalmente relatan correctamente cuatro o cinco letras. Esta amplitud o limitación no se debe a la capacidad perceptual del sujeto, sino que viene impuesta por su sistema de memoria (Sperling, 1960). A partir de los trabajos de Sperling $(1960,1967)$ se asume que toda la información presentada visualmente permanece disponible para su procesamiento durante varios cientos de milisegundos después que termina la exposición. Los elementos de la información presentada son entonces explorados (scaned) secuencialmente por un procesador central. Todo elemento que recibe la atención del explorador pasa a la siguiente fase de procesamiento, pero si la excitación neural de un elemento se desvanece antes de ser explorado por el scanner, la información asociada a tal excitación se pierde (ver Ruiz Vargas, 1980, para una revisión).

Neale, McIntyre, Fox y Cromwell (1969) examinaron la ejecución de pacientes esquizofrénicos y sujetos normales en una

(") Este articulo forma parte de la Tesis Doctoral del autor, que, bajo el título Memoria icónica y esquizofnenia: Fases iniciales de procesamiento y déficit cognitivo, fue presentada y juzgada en la Universidad Autónoma de Madrid (1979). 
tarea de amplitud de aprehensión, urilizando la técnica del informe parcial de Estes (1965) que sólo requiere reconocimiento. Encontraron que la amplitud de los esquizofrénicos era aproximadamente la mitad de la de los normales e interpretaron tal reducción como resultado de una incapacidad para procesar eficientemente la información. Sin embargo, un estudio posterior de Cash, Neale y Cromwell (1972) demostró que los esquizofrénicos procesan la información al mismo ritmo que los normales. Estos autores encontraron que en una tarea de amplitud de aprehensión, según la técnica del informe total (la cual requiere reconocimiento y recuerdo), la ejecución de los esquizofrénicos era tan buena como la de los normales. Por su parte, Spohn, Thetford y Woodham (1970) midieron la amplitud de aprehensión de esquizofrénicos y normales introduciendo como variable independiente la duración de la exposición. Comprobaron que en cualquier condición $(50,250,750,1.100 \mathrm{mseg}$. de exposición) el rendimiento esquizofrénico era inferior al de los sujetos normales. Estos autores atribuyeron tales resultados a una tasa de lectura del material visual relativamente inferior o a una incapacidad, por parte del paciente esquizofrénico, para beneficiarse del incremento del tiempo de lectura ofrecido por la imagen visual de mayor duración. Esta interpretación está en clara concordancia con la de Neale et al. (1969), ya que ambos estudios parecen apoyar la idea de una tasa lenta de procesamiento en los pacientes esquizofrénicos. Además, ambas posturas son consistentes con la teoría de Yates $(1966,1973)$-que atribuye el déficit esquizofrénico a un procesamiento de la información anormalmente lento-, aunque experimentalmente no hayan comprobado si el variar la tasa de presentación modifica la ejecución esquizofrénica, como parece ser la consecuencia básica derivada de tal teoría.

Nuestro trabajo experimental sobre amplitud de aprehensión ha tenido en cuenta dos variables independientes, la duración de la exposición y el número de items presentados. Se trata de replicar los tres estudios anteriores y de un modo especial el de Spohn et al. (1970), así como la teoría de Yates $(1966,1973)$. Consideramos que la tasa de presentación viene determinada al menos por dos componentes: uno temporal y otro relativo a la cantidad de información expuesta. Esto es, se puede variar la tasa de presentación aumentando o disminuyendo el tiempo de exposición mieritras se mantiene constante la cantidad de información presentada, o bien, aumentando o disminuyendo la cantidad de información mientras se mantiene constante la duración de la exposición.

\section{METODO}

1. Sujetos. El experimento fue realizado con un total de 30 sujetos (20 pacientes ingresados en el Hospital Psiquiátrico Alonso Vega, de Madrid, diagnosticados de esquizofrénicos, y 10 sujetos normales). Se establecieron los siguientes grupos: a) 11 esquizofrénicos paranoides (9 mujeres y 2 hombres), que según la longitud de su hospitalización se repartían entre $\mathbf{4}$ crónicos (duración de la enfermedad de cuatro años o más desde el primer indicio claro de psicosis) y 7 agudos (pacientes con duración de la enfermedad inferior a los cuatro años). Asimismo, atendiendo a su ajuste premórbido, los 11 esquizofrénicos paranoides se dividían en 5 esquizofrénicos procesales y 6 esquizofrénicos reactivos (esta dimensión fue establecida aplicando al historial clínico de cada paciente la escala de Phillips). b) 9 esquizofrénicos no-paranoides (8 mujeres y 1 hombre) repartidos, según la longitud de su hospitalización, en 5 esquizofrénicos crónicos y 4 esquizofrénicos agudos y, según su ajuste premórbido, en 7 esquizofrénicos procesales y 2 esquizofrénicos reactivos. c) 10 sujetos normales de control, todos ellos mujeres integrantes del equipo médico (ayudantes téc- 
nicos sanitarios) y del personal administrativo, con edades comprendidas entre 23 y 36 años.

Los pacientes esquizofrénicos fueron seleccionados atendiendo al diagnóstico psiquiátrico de esquizofrenia ya establecido, haciendo especial hincapié en que no existiera ninguna evidencia de organicidad, retraso mental, adicción o alcoholismo. Teniendo en cuenta que el material estimular estaba formado por letras, fue imprescindible seleccionar atendiendo al nivel cultural. El nivel educativo iba desde la enseñanza primaria (4 pacientes) hasta algún curso universitario (1 paciente), pasando por bachillerato elemental (8 pacientes) y secretariado. La edad oscilaba entre 17 y 43 años.

2. Aparato. La presentación de los estímulos se hizo con un taquistoscopio de cuatro canales TKK 226 (Tipo B) de la firma Takei Kiki Kogyo (Japón). Este aparato permite programar tiempos de exposición con un rango de medida que oscila entte $0,0001 \mathrm{seg}$. y $9.999 \mathrm{seg}$. Dispone de tres campos visuales, cada uno con un tamaño aproximado de 200 por $200 \mathrm{~mm}$. iluminado indirectamente por dos lámparas fluorescentes de $4 \mathrm{~W}$. La distancia desde el campo visual al ojo del observador es de $800 \mathrm{~mm}$. aproximadamente.
3. Material. Se construyeron sesenta tarjetas-estímulo diferentes que se agruparon en seis conjuntos distintos de diez tarjetas cada uno. La diferencia entre los conjuntos era cuantitativa, es decir, se diferenciaban entre sí por el número de letras consonantes que contenían. El conjunto con menor número de consonantes contenía tres y el mayor ocho. Los estímulos fueron construidos adhiriendo Letraset Instant Lettering (modelo 141) de color negro sobre tarjetas de cartón blanco. El tamaño de cada item era de $30 \mathrm{~mm}$. de ancho por $25 \mathrm{~mm}$. de alto, subtendiendo un ángulo visual de aproximadamente $2,15^{\circ}$ horizontalmente por $1,8^{\circ}$ verticalmente. El tamaño de cada conjunto de items aparece especificado en el cuadro 1. La primera cifra corresponde a la anchura o a la horizontalidad y la segunda a la altura o verticalidad, según se trate de superficie o ángulo visual, respectivamente. Para la construcción de cada tarjeta se tuvieron en cuenta las siguientes restricciones: a) no se utilizaron vocales; b) se prescindió de las consonantes $\tilde{\mathrm{N}}, \mathrm{W}, \mathrm{X}, \mathrm{T}$; c) una misma letra no aparecía más de una vez en cada tarjeta, y d) dentro de cada conjunto, una misma letra sólo ocupaba una vez el mismo emplazamiento.

4. Procedimiento. El campo 1 del taquistoscopio se utilizó para presentar un

CUADRO 1. Tamaño y ángulo visual subtenso por cada conjunto de torjetas-estímulo

\begin{tabular}{|c|c|c|}
\hline$N .^{\circ}$ de items & Superficie & \multicolumn{1}{|c|}{ Angulo visual } \\
\hline 3 & $160 \mathrm{~mm}$. por $30 \mathrm{~mm}$. & $11,3^{\circ}$ por $2.15^{\circ}$ \\
\hline 4 & $180 \mathrm{~mm}$. por $30 \mathrm{~mm}$. & $12,7^{\circ}$ por $2,15^{\circ}$ \\
\hline 5 & $160 \mathrm{~mm}$. por $90 \mathrm{~mm}$. & $11,3^{\circ}$ por $6,4^{\circ}$ \\
\hline 6 & $160 \mathrm{~mm}$. por $90 \mathrm{~mm}$. & $11,3^{\circ}$ por $6,4^{\circ}$ \\
\hline 7 & $180 \mathrm{~mm}$. por $90 \mathrm{~mm}$. & $12,7^{\circ}$ por $6,4^{\circ}$ \\
\hline 8 & $180 \mathrm{~mm}$. por $90 \mathrm{~mm}$. & $12,7^{\circ}$ por $6,4^{\circ}$ \\
\hline
\end{tabular}




\section{Estudios}

pequeño punto negro de fijación de unos $10 \mathrm{~mm}$. de diámetro. Este punto de fijación coincidía con el centro del campo visual. Las tarjetas-estímulo fueron presentadas en el campo 2, siguiendo el procedimiento siguiente: tras una breve explicaciôn del aparato y del objetivo de la investigación, se le pedía al sujeto que se sentase en el lugar adecuado y fijase su cabe$\mathrm{za}$ de tal manera que sus ojos pudieran enfocar perfecta y totalmente el campo visual para la presentación de los estímulos. El sujeto era entonces instruido para que informara en alta voz de todas las letras que pudiese, inmediatamente después de la presentación, sin preocuparse por el orden o emplazamiento de los items. Estas instrucciones eran seguidas por 30 ensayos de práctica ( 5 con cada conjunto de tarjetas), tras los cuales se le preguntaba si había entendido la tarea.

Si no surgía ninguna cuestión, cada sujeto recibía a continuación un total de 300 presentaciones ordenadas en cinco bloques. Cada bloque correspondía a un tiempo de exposición distinto, a saber: 15, 50, $100,500 \mathrm{mseg}$. Para balancear los resultados del grupo, unas veces la presentación era ascendente y otras descendente, según el tiempo de exposición. Esto es, unos sujetos empezaban el experimento con exposiciones ascendentes desde $15 \mathrm{mseg}$. y otros con exposiciones descendentes desde $500 \mathrm{mseg}$. Los seis conjuntos de tarjetas-estímulo ( $3,4,5,6,7$ y 8 items) eran presentadas ordenadamente, bien en orden ascendente o descendente. Puesto que cada conjunto estaba compuesto por 10 tarjetas-estímulo el total de presentaciones por bloque era de 60.

Las presentaciones eran iniciadas por el experimentador con la pregunta « $i$ Preparado? e iban separadas por un pequeño intervalo cercano a los diez segundos; entre la presentación de cada conjunto de tarjetas transcurría un tiempo aproximado a los dos minutos, y entre cada bloque de ensayos se daba un descanso cercano a los cinco minutos. La duración media de cada bloque de ensayos parece en el cuadro 2 . La duración total del experimento osciló entre 120-180 minutos, que normalmente se repartieron en dos o tres jornadas para evitar la fatiga de los sujetos.

Los informes de los sujetos eran registrados por el experimentador en unas hojas preparadas al efecto. La recogida de los datos intentaba ser una copia literal del relato del sujeto: letras informadas, orden, repeticiones, errores, etc.

\section{RESULTADOS}

El efecto del tamaño de la exposición (desde tres a ocho items) y de las distintas condiciones de la duración de la exposición (desde 15 a $500 \mathrm{mseg}$.) fue evaluado con un análisis de varianza aplicảndo el diseño factorial con medidas repetidas

CUADRO 2. Duraciones medias de los distintos bloques de ensayos

\begin{tabular}{|c|c|}
\hline Tiempo de exposición & Duración del bloque \\
\hline $15 \mathrm{mseg}$. & 15 minutos \\
\hline $50 \mathrm{mseg}$. & 18 minutos \\
\hline $100 \mathrm{mseg}$. & 20 minutos \\
\hline $200 \mathrm{mseg}$. & 22 minutos \\
\hline $500 \mathrm{mseg}$. & 25 minutos \\
\hline
\end{tabular}


CUADRO 3. Anólisis de varianza

\begin{tabular}{|l|r|r|r|r|r|}
\hline & \multicolumn{1}{|c|}{$S C$} & \multicolumn{1}{c|}{ gl } & \multicolumn{1}{c|}{$M C$} & \multicolumn{1}{c|}{$F$} & \multicolumn{1}{c|}{$p<$} \\
\hline Media & \multicolumn{1}{|c|}{6967.46716} & 1 & 6967.46716 & 1819.73822 & .000 \\
J (variable & & & & & \\
de grupo) & 282.74440 & 8 & 35.34305 & 9.23077 & .000 \\
Error & 80.40542 & 21 & 3.82883 & & \\
Exposición (E) & 16.84653 & 4 & 4.21163 & 21.19585 & .000 \\
EJ & 8.40617 & 32 & .26269 & 1.32205 & .157 ns \\
Error & 16.69087 & 84 & .19870 & & .000 \\
Tamaño (T) & 73.23303 & 5 & 14.64661 & 59.56060 & .000 \\
TJ & 75.03719 & 40 & 1.87593 & 7.62849 & \\
Error & 25.82065 & 105 & .24591 & & .000 \\
ET & 289.51189 & 20 & 14.47559 & 222.53520 & .000 \\
ETJ & 27.63586 & 160 & .17272 & 2.65531 & \\
Error & 27.32040 & 420 & .06505 & & \\
\hline
\end{tabular}

BMDP2V. Los resultados aparecen en el cuadro 3.

Las diferencias en amplitud de aprehensión entre normales y esquizofrénicos (paránoides y no-paranoides) (1) fueron altamente significativas. Sólo el efecto cruzado del grupo $(J)$ con el tiempo de exposición (E) resultó no significativo. Para valorar el efecto intragrupal de la variación de la tasa de presentación fueron calculadas pruebas $t$ para muestras correlacionadas siguiendo el procedimiento siguiente: a) para evaluar el efecto del tiempo de exposición, manteniendo constante el número de items, se calculó la prueba $t$ entre las medidas de cada grupo con $15 \mathrm{mseg}$. de exposición y el resto de las condiciones de duración $(50,100,200,500 \mathrm{mseg}$.), manteniendo constante la cantidad de información: 8 items. El cuadro 4 muestra los distintos valores de $t$ en cada una de las condiciones comparadas. El efecto del tiempo sobre el rendimiento de los sujetos normales sólo fue significativo en la condición de $500 \mathrm{mseg}$. Los esquizofrénicos paranoicos se beneficiaron significativamente del tiempo de exposición a partir de los 200 mseg. El grupo de los esquizofrénicos noparanoicos, sin embargo, no mejoró su rendimiento en ninguna condición. En la Figura 1 se muestra el rendimiento de los tres grupos como. una función de la duración de la exposición.

A pesar de que gráficamente se advierte cierta diferencia entre esquizofrénicos paranoicos y esquizofrénicos no-paranoicos, calculada la prueta $t$ no aparecieron diferencias significativas entre ambos grupos de pacientes (ver cuadro 5). b) El efecto del tamaño de la exposición, esto es, la cantidad de información, mientras se mantenía constante la duración de la misma, se va. loró calculando la prueba $t$ entre los rendimientos promedios con 6 items y los valores obtenidos con 7 y 8 items. El tiempo elegido fue $100 \mathrm{mseg}$. por considerarlo el más usual en estos tipos de investigaciones (tal duración sólo permite una fijación ocular). Los rendimientos con tamaños inferiores a 6 items no se han considerado por estar, en gran parte de los casos, por debajo de la propia amplitud de aprehen-

(1) Ante la imposibilidad de considerar las tres dimensiones citadas en una interacción total, como proponen Chapman y Chapman (1973) (de los ocho subgrupos resultantes tres de ellos - paranoide reactivo crónico, no paranoide reactivo crónico y no paranoide reactivo agudo- sólo contaban con un sujeto), optamos por la dimensión paranoide versus no-paranoide, considerada por algunos autores la de mayor fiabilidad (Lang y Buss, 1965). 


\section{Estudios}

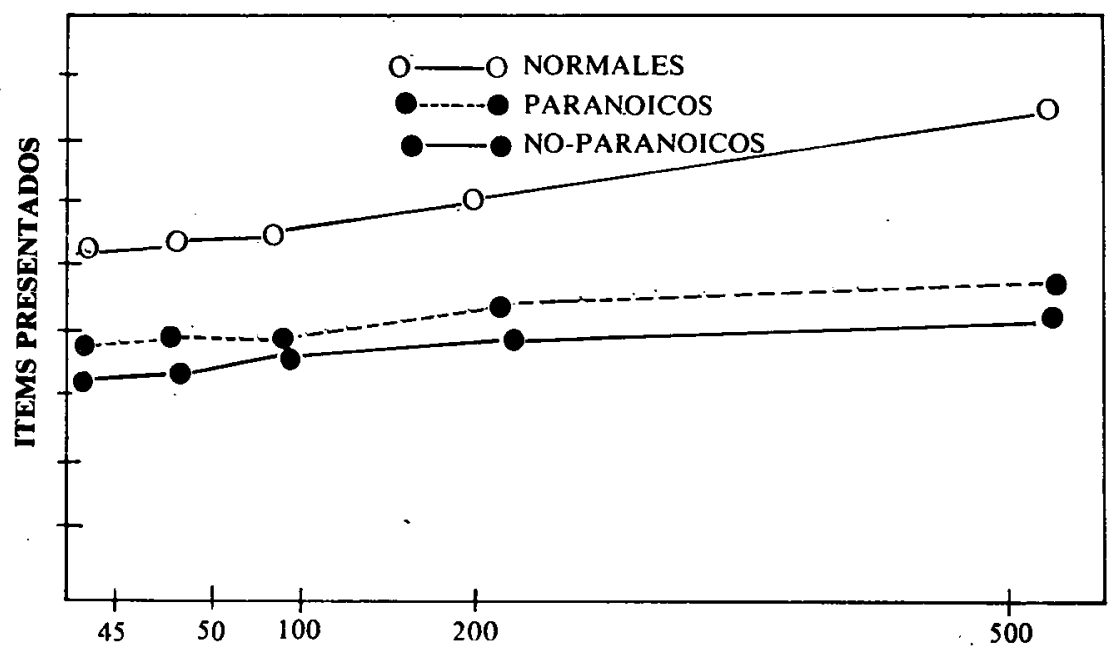

Figura 1 Duración de la exposición (msg.)

Rendimiento de los tres grupos como una función de la duración de la exposición

CUADRO 4. Valores t para el efecto de la duración de la exposición manteniendo constante la cantidad de información (8 items)

\begin{tabular}{|l|c|c|c|c|c|c|}
\hline & $50 \mathrm{mseg}$ & $100 \mathrm{mseg}$ & $200 \mathrm{mseg}$ & $500 \mathrm{mseg}$ & $\mathrm{gl}$ & $t^{*}$ \\
\hline Normales & 0,480 & 0,958 & 1,571 & 5,473 & 9 & 2,262 \\
\hline E. paranoicos & 0,416 & 0,358 & 4,137 & 3,070 & 10 & 2,228 \\
\hline E. no-paranoicos & 0,677 & 0,261 & 2,225 & 0,320 & 8 & 2,306 \\
\hline
\end{tabular}

Cada celdilla indica el valor de $t$ entre el rendimiento promedio con $15 \mathrm{mseg}$ de exposición y el indicado en cada columna. $\mathrm{t}^{*}=$ valores de las tablas con un nivel de confianza del $5 \%$.

TABLA 5. Valores t entre el rendimiento de esquizofrénicos paranoicos y esquizofrénicos no-paranoicos en función del tiempo de exposición

\begin{tabular}{|c|c|c|c|c|c|c|}
\hline $15 \mathrm{mseg}$ & $50 \mathrm{mseg}$ & $100 \mathrm{mseg}$ & $200 \mathrm{mseg}$ & $500 \mathrm{mseg}$ & $\mathrm{gl}$ & $t^{*}$ \\
\hline 1,428 & 1,468 & 0,519 & 1,082 & 0,653 & 18 & 2,101 \\
\hline
\end{tabular}

$t^{*}=$ valor de las tablas con un nivel de confianza del $5 \%$.

sión, como consecuencia misma del bajo número de items presentados. El cuadro 6 muestra los distintos valores de $t$ en cada una de las condiciones comparadas. Ningún grupo acusó de forma significativa el efecto de la variación de la cantidad de información presentadà. La Figura 2 muestra de manera gráfica los distintos rendimientos promedios como una función del número de items presentados. Aunque las 
TABLA 6. Valores t para el efecto de la cantidad de información presentada manteniendo constante el tiempo de exposición (100 mseg)

\begin{tabular}{|l|c|c|c|c|}
\hline & 7 items & 8 items & gl & $t^{*}$ \\
\hline Normales & 0,139 & 1,948 & 9 & 2,262 \\
\hline E. paranoicos & 0,171 & 0,301 & 10 & 2,228 \\
\hline E. no-paranoicos & 0,165 & 0,912 & 8 & 2,306 \\
\hline
\end{tabular}

Cada celdilla indica el valor de $t$ entre el rendimiento promedio con 6 items y el $\cdot$ indicado en cada columna. $t^{*}=$ valores de las tablas con un nivel de confianza del $5 \%$.

\section{Figura 2}

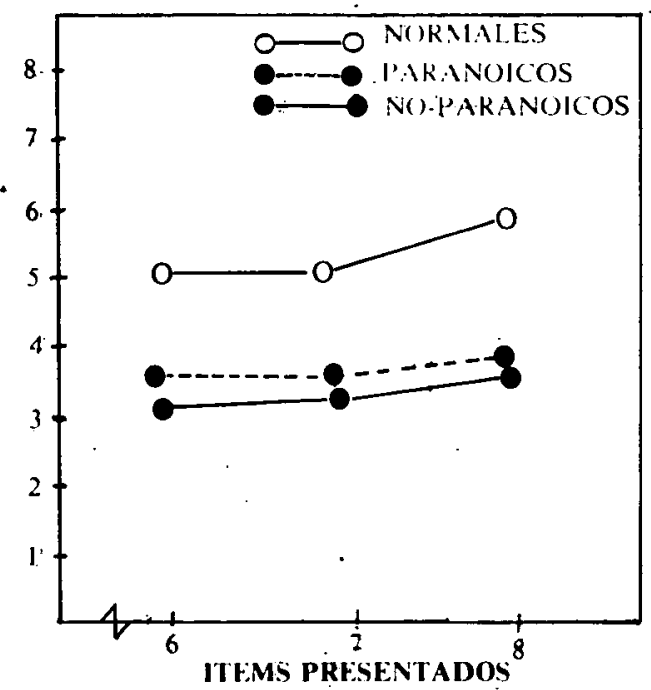

Rendimiento de los tres grupos como una función del número de items

gráficas sean ascendentes en los tres grupos, en ningún caso fueron significativas las diferencias intragrupales. La superioridad de los esquizofrénicos paranoides sobre los esquizofrénicos no-paranoides fue $\tan$ pequeña que estadísticamente no resultó significativa.

\section{DISCUSION}

Los resultados de este experimento indican que los esquizofrénicos - paranoicos y no-paranoicos- procesan la información visual menos eficientemente que los sujetos normales. Concretamente, los esquizofrénicos tienen una amplitud de aprehensión reducida. Estos resultados son consistentes con los de Neale, McIntyre, Fox y.
Cromwell (1969), quienes encontraron que el rendimiento de los pacientes esquizofrénicos en tareas de amplitud de aprehensión era aproximadamente la mitad del de los sujetos normales. No obstante, hay que reseñar que la técnica utilizada por Neale et al. (1969) difiere sensiblemente de la empleada por nosotros. Aquellos autores hicieron uso de la técnica del informe parcial de Estes (1965), que sólo implica reconocimiento, mientras que nosotros hemos utilizado la técnica del informe total que requiere reconocimiento y recuerdo. Con esta misma técnica, Cash Neale y Cromwell (1972) no encontraron diferencias significativas entre sujetos normales y pacientes esquizofrénicos. Sin embargo, Spohn, Thetford y Woodham (1970), con 
una técnica y un material muy similar al nuestro, también encontraron diferencias significativas entre normales y esquizofrénicos.

El hecho de que Cash et al. (1972) hayan concluido que no existe déficit esquizofrénico en amplitud de aprehensión puede deberse a un error metodológico. De los dos tipos de tarjetas que utilizaron, uno sólo contenía 4 letras y el otro 8 . En efecto, existe una gran probabilidad de que con los estímulos de cuatro letras el rendimiento de los pacientes esquizofrénicos no mostrara déficit alguno con respecto al de los sujetos normales, ya que la ejecución máxima no podía superar nunca el valor 4 para todos los sujetos. La falta, pues, de diferencias entre los dos grupos pudo deberse a un refecto-techos. La misma falta de control ha sido encontrada por Chapman y Chapman (1978) en el experimento de Neale et al. (1969). Consideramos pues que, en situaciones experimentales bien controladas, la amplitud de aprehensión de los esquizofrénicos es significativamente inferior a la de los sujetos normales. Al mismo tiempo, podemos afirmar que entre los subgrupos esquizofrénicos (paranoicos, no-paranoicos) no existen diferencias significativas. Este último hallazgo es consistente con los resultados de Spohn et al. (1970), quienes no encontraron diferencias significativas entre ningún subgrupo de esquizofrénicos de las tres dimensiones controladas (paranoico versus no-paranoico, agudo versus crónico, reactivo versus procesal).

El efecto de la duración de la exposición tuvo cierta significación sobre el rendimiento de los sujetos normales y esquizofrénicos paranoicos. Los estudios sobre este parámetro estimular, con sujetos normales, no parecen indicar que la duración de la exposición tenga una influencia significativa sobre el rendimiento (Sperling, 1960 , experimento 2). Si bien Mackworth (1963, experimento 1) encontró que, al menos hasta los primeros 50 milisegundos. la exactitud del informe mejoraba al in- crementar la duración de la exposición, las críticas metodológicas de su trabajo parecen invalidar sus hallazgos. Evidentemente, toda investigación está siempre abierta a la crítica, sin embargo, consideramos que nuestro experimento no adolece de ningún fallo metodológico que pueda explicar que los sujetos normales tengan un rendimiento significativamente superior a partir de los 500 mseg. y que los esquizofrénicos paranoicos presenten dicha mejoría desde los 200 mseg. Podrían imputarse tales resultados a la variable aprendizaje, por entender que la condición de $500 \mathrm{mseg}$. iría siempre precedida por el resto; sin embargo, y como ya se ha explicado en el Procedimiento, para anular tales efectos aproximadamente la mitad de los sujetos iniciaban al bloque de ensayos por un extremo (e. g. 15 mesg.) y la otra mitad por el otro (e. g. $500 \mathrm{mseg}$.). Puesto que el efecto también se deja sentir en los sujetos normales, su interpretación tal vez caiga fuera de los objetivos de este estudio, al tiempo que requerirá nuevas investigaciones más precisas si cabe. Es posible que sea más interesante para nosotros tratar de averiguar por qué los esquizofrénicos no-paranoicos no mejoraron su rendimiento con el incremento del tiempo de exposición. Podemos razonar tal hallazgo como debido a una tasa de lectura del material visual inferior a la de los esquizofrénicos paranoicos. En toda tarea de búsqueda, como es la lectura, el ojo está sometido a una serie de movimientos sacádicos que tienen como objetivo colocar las distintas partes del estímulo sobre la fóvea. Un movimiento sacádico se produce cada 200-250 mseg., por lo tanto, sólo en la condición de $500 \mathrm{mseg}$. de exposición era posible más de una fijación ocular. Con esto no tratamos de decir que la am. plitud de aprehensión esté determinada por el sistema visual — Sperling (1960) puso de manifiesto experimentalmente que tal limitación viene impuesta por el sistema de memoria-, pero sí es posible afirmar que el bajo rendimiento de los esqui- 
zofrénicos esté en parte determinado por su baja tasa de movilidad ocular, y de una manera más acusada en los esquizofrénicos no-paranoides. Esta interpretación es consistente con los estudios sobre reactividad pupilar (Hakerem y Lidsky, 1975) y movimientos oculares (Holzman et al., 1974; Acker y Toone, 1978) que han puesto de manifiesto las diferencias significativas existentes entre sujetos normales y esquizofrénicos.

Por otra parte, el variar la cantidad de información no tuvo ningún efecto sobre el rendimiento de ninguno de los grupos. Pudo apreciarse en todos los sujetos cierta mejoría a medida que aumentaba el número de items, pero las diferencias no fueron significativas. Podemos afirmar entonces que el rendimiento no depende del número de items presentado.

Los resultados obtenidos con los sujetos esquizofrénicos al variar la tasa de presentación - a nivel de tiempo de exposición y cantidad de información- no parecen ser consistentes con la teoría de Yates (1966, 1973), que postula la existencia de un procesador central anormalmente lento como responsable del déficit esquizofrénico. De tal teoría se deriva la hipótesis de que al disminuir la tasa de presentación del input el rendimiento esquizofrénico se hace tan eficaz como el de los normales (Chapman y MacGhie, 1963; Payne, Hochberg y Hawks, 1970; Wishner y Wahl, 1974; Wijesinghe, 1977); sin embargo, en nuestro estudio la variación de la tasa de presentación, concretada en un enientecimiento de las presentaciones (desde 15 rnseg. a 500 mseg.) y en una disminución de la cantidad de información (desde 8 items a 3 items), no supuso un cambio significativo en el rendimiento esquizofrénico. Sólo a partir de los $200 \mathrm{mseg}$. y únicamente en los esquizofrénicos paranoides, aparecieron resultados que apoyan relativamente la hipótesis de Yates; no obstante, el cambio sufrido por la tasa de pre. sentación al variar el número de items no modificó en absoluto el rendimiento de los pacientes esquizofrénicos. Así pues, la idea de un procesador central que no funciona lo bastante rápido no parece recibir demasiado apoyo con nuestros resultados, ya que, en términos generales, podemos afirmar que tanto si la tasa de presentación era rápida o lenta y mayor o menor, el rendimiento esquizofrénico en amplitud de aprehensión fue significativamente más bajo que el normal.

Como ya se ha dicho, la respuesta de amplitud implica procesos atencionales, cognitivos y mnésicos; sin embargo, y a la vista de los datos disponibles, el déficit encontrado por nosotros en los pacientes esquizofrénicos sólo puede ser interpretado como debido a un procesamiento ineficaz de la información. El análisis e interpretación de un nuevo grupo de experimentos, realizados por el autor, tratarán de concretar qué proceso o fase del procesamiento de la información es el responsable del déficit cognitivo esquizofrénico.

\section{Referencias}

ACKER, W. y TOONE, B. (1978): Attention, eye tracking and schizophrenia. British Journal of Social and Clinical Psychology, 17, 173-182.

CASH, T.; NEALE, J. M., Y CROMWELL, R. L. (1972): Span of apprehension in schizophrenia: Full-report technique. Journal of Abnormal Psychology, 79, 322-327.

CHAPMAN, L. J., y CHAPMAN, J. P. (1973): Disordered thought in schizophrenia. Englewood Cliffs, N. J.: Prentice-Hall.

CHAPMAN, L. J., y CHAPMAN, J. P. (1978): The measurement of differential deficit. Journal of Psychiatric Research, 14, 303-312.

CHAPMAN, J., y McGHIE, A. (1963): An approach to the psychotherapy of cognitive dysfunction. British Journal of Medical Psychology, 36, 253-260.

ESTES, W. K. (1965): A technique for assessing variability of perceptual span. Proceedings of the National Academy of Science, 54, 403-407. 
HAKEREM, G., y LIDSKY, A. (1975): Characteristics of pupillary reactivity in psychiatric patients and normal controls. En M. L. KIETZMAN, S. SUTTON y J. ZUBIN (Eds.): Experimental approaches to psychopathok ayy. Londres. Academic Press.

HAWKS, D. V., Y MARSHALL, W. L. (1971): A parsimonious theory of overinclusive thinking and retardation in schizophrenia. British Journol of Medical Psychology, 44, 75-83.

HOLZMAN, P. S.; PROCTOR, L. R.; LEVY, D. L.; YASILLO, N. J.; MELTZER, H. Y., y HURT, S. W. (1974): Eye-tracking dysfunctions in schizophrenic patients and their relatives. Archives of General Psychiatry, 31, 143-151.

LANG, P. J., y BUSS, A. H. (1965): Psychological deficit in schizophrenia. Il. Interference and activation. Journal of Abnormal Psychology, 70, 77-106.

LAWSON, J. S.; MCGHIE, A., y CHAPMAN, J. (1967): Distractibility in schizophrenia and organic cerebral disease. British Journal of Psychiatry, 113, 527-535.

MACKWORTH, J. F. (1963): The duration of visual image. Canadian Journal of Psychology, 17, 62-81.

McGHIE, A. (1966): Psychological studies of schizophrenia. British Journal of Medical Psychology, 39, 281-288.

NEALE, J. M.; MCINTYRE, C. W.; FOX, R., y CROMWELL. R. L. (1969): Span of apprehension in acute schizophrenics. Journal of Abnormal Psychology, 74, 593-596.

PAYNE, R. W. (1960): Cognitive abnormalities. En EYSENCK, H. J. (Ed.): Handbook of Abnormal Psychology. Nueva York: Basic Books.

PAYNE, R. W.; HOCHBERG, A. C., y HAWKS, D. V. (1970): Dichotic stimulation as a method of assessing disorder of attention in overinclusive schizophrenic patients. Journal of Abnormal Psychology, 76, 185-193.

PHILLIPS, L. (1953): Case history data and prognosis in schizophrenia. Journal of Nervous and Mental Disease, 117, 515-525.

RUIZ VARGAS, J. M. (1979): Memoria icónica y esquizofrenia: Fases iniciales de procesamiento $y$ déficit coqnitivo. Tesis Doctoral no publicada. Universidad Autónoma de Madrid.

RUIZ VARGAS, J. M. (1980): La memoria icónica: Una revisión. Revista de Psicología General y Aplicada, 35 (en prensa).

SPERLING, G. (1960): The information available in brief visual presentations. Psychological Monographs, 74 (Whole n. ${ }^{\circ} 498$ ).

SPERLING, G. (1967): Successive approximations to a model for short-term memory. Acto Psychological, 27, 285-292.

SPOHN, H. E.; THETFORD, P. E., y WOODHAM, F. L. (1970): Span of apprehension and arousal in schizophrenia. Journal of Abnormal Psychology, 75, 113-123.

WIKESINGHE, O. B. A. (1977): The effect of varying the rate of presentation on the information transmission of schizophrenic and control groups. British Journal of Psychiatry, 130, 509-513.

WISHNER, J., y WAHL, O. (1974): Dichotic listening in schizophrenia. Journal of Consulting and Clinicol Psychology, 42, 538-546.

YATES, A. J. (1966): Data processing levels and thought disorder in schizophrenia. Australian Journal of Psychiatry, 18, 103-117.

YATES, A. J. (1973): Terapia del comportamiento. México: Trillas. (Edición original, Nueva York, Wiley, 1970).

\section{Resumen}

Esta investigación ha tenido como objetivo comparar la amplitud de aprehensión de dos grupos de pacientes esquizofrénicos (paranoide y no-paranoide) y la de un grupo de sujetos normales. Las variables independientes han sido la duración de la exposición y el número de items. Los resultados sugieren que los esquizofrénicos procesan la información visual menos eficientemente que los normales.

\section{Summary}

The aim of this research has been to compare the span of apprehension for two groups of schisophrenic patients (paranoid and non-paranoid) with that for a normal group. The duration of exposure and the number of items have been treated as independent variables. The results suggest schizophrenic patients process visual information less efficiently than normal subjects.

\section{Resumé}

Cette recherche essaie de compatet l'amplitude d'apréhension de deux groupes des patients schizophrènes (paranoïaque et non-paranoïaque) avec cela d'un groupe de sujets normales. Etant les variables independants la durèe de l'exposition et le quantité des items. Les resultats suggerent que les schizophrénes font le processus de l'information visuel moins efficientement que les sujets normales. 